\title{
Rare Cases in ENT
}

\author{
Kalyan Pal, ${ }^{1}$ Dipanjan Chakraborty, ${ }^{1}$ Sohag Kundu, ${ }^{1}$ Subrata Mukhopadhyay ${ }^{1}$
}

\section{ABSTRACT}

In our day to day ENT practice we commonly come across diseases involving the larynx, the oral cavity and the paranasal sinuses. These range from inflammatory disorders to benign and malignant neoplasms. Carcinomas involving the head and neck region are most commonly squamous cell carcinoma. However, a small proportion of cases present with other variants of carcinoma or infective pathology uncommon for the site. In this study we present three rare cases encountered in the out-patient department, namely, Primary Malignant Melanoma of the larynx, Neuroendocrine Tumor of the nose and paranasal sinuses and Rhinosporidiosis of cheek.

Keywords:

Melanoma; Carcinoma, Neuroendocrine; Rhinosporidiosis

$\mathrm{P}$ rimary mucosal melanomas arise from melanocytes located in mucous membranes lining respiratory, gastrointestinal and urogenital tracts. Malignant melanoma of the larynx is a rare cancer that can appear as a primary tumor or as a metastasis from a cutaneous head and neck primary lesion. ${ }^{1}$ Melanocytes have phagocytic and possibly antigen-presenting function, and also produce various cytokines., ${ }^{2,3}$ Majority of laryngeal melanomas involve the supraglottic larynx. Less common sites include the glottis. The least common site is the subglottis. In this article, we report a rare case of primary malignant melanoma of the supraglottic larynx.

Small cell neuroendocrine carcinoma of nose and paranasal sinuses have been reported in literature, with a male female ratio of 1.6:1 and mean age of 51 years. Authors rely on the Kadish staging system of nasal cavity and paranasal sinus tumors (Kadish A: limited to nasal cavity, Kadish B: limited to nasal cavity and paranasal sinuses, Kadish C: tumour extending beyond the nasal cavity and paranasal sinuses). ${ }^{4,5}$ Tumours present with a variety of different histological patterns, including cords, sheets, ribbons, pseudoglands and rosette formations. The degree of cellular pleomorphism, mitotic activity, and necrosis increases as the tumor becomes more poorly

1 - Department of ENT, Medical College, Kolkata

\section{Corresponding author:}

Dr Kalyan Pal

email: kalyan.pal71@gmail.com differentiated (small cell carcinoma). In this report we present a 45 year old female presenting with a sinonasal mass diagnosed as neuroendocrine carcinoma.

Rhinosporidiosis is a benign chronic granulomatous disease caused by Rhinosporidium seeberi. The most common site of infection in human is the nose, ${ }^{6}$ cheek being a rare site. Rhinosporidiosis in the parotid duct has also been reported. ${ }^{7}$ The most common mode of spread to host is transepithelial infection or autoinoculation. Individuals probably acquire the disease from water contaminated by diseased cattle. In this paper we present one such case presenting with a right sided cheek swelling diagnosed with rhinosporidiosis.

\section{Case 1: Primary Malignant Melanoma of the larynx}

A 45 year old male presented with history of foreign body sensation in the throat and difficulty in deglutition for 1 month. There was no history of hoarseness, dysphagia, odynophagia or otalgia. He presented to the emergency with stridor. Indirect Laryngoscopic examination revealed a smooth blackish mass arising from the laryngeal surface of epiglottis and left aryepiglottic fold, occluding the whole of the laryngeal inlet. (Fig. 1) No other endolaryngeal structures were visible. No neck nodes were palpable. An emergency tracheostomy was performed under local anaesthesia to relieve respiratory distress.

Assessment by Fibreoptic Laryngoscopy showed the mass to be arising from the laryngeal surface of epiglottis 


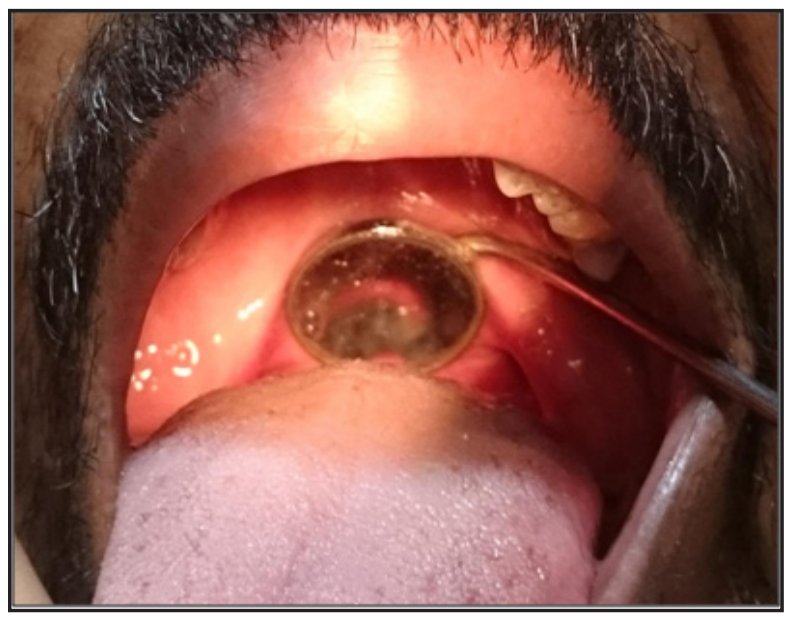

Fig.1. Blackish mass involving laryngeal surface of epiglottis on indirect laryngoscopy

and left aryepiglottic fold, occluding the whole of the laryngeal inlet and obscuring view of endolaryngeal structures. (Fig. 2) CT scan was done to assess extent of the lesion. (Fig. 3) Retrograde endoscopy through the tracheostoma showed no involvement of glottis, subglottis. The trachea was free upto the level of carina. The patient subsequently underwent Direct Layngoscopy under general anaesthesia. The findings of Direct Laryngoscopy were corroborative with those of Fibreoptic Larynygoscopy. Biopsy was taken from the mass and sent for HPE. A paraffin section stained with hematoxylin and eosin (H\&E) showed tumour cells with clear nuclei exhibiting pleomorphism and melanin pigmentation in the cytoplasm. These cells

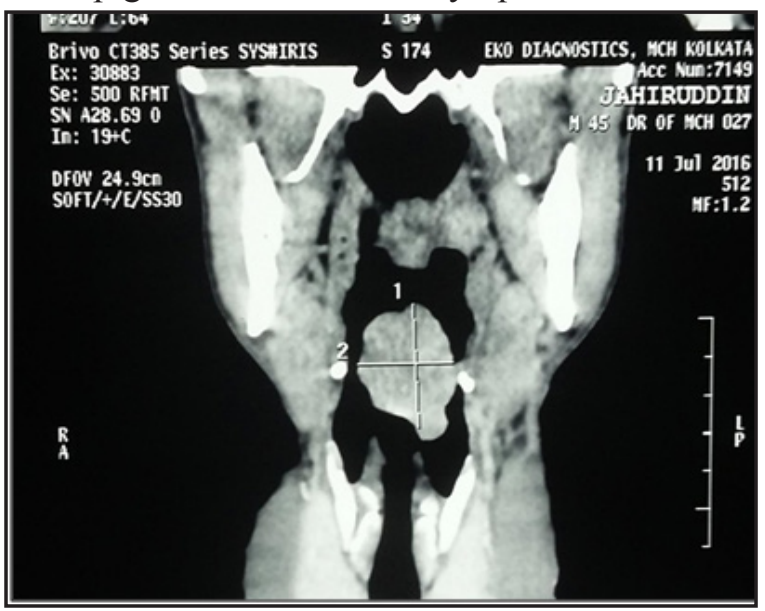

Fig. 3. Coronal CT with contrast showing extent of the mass

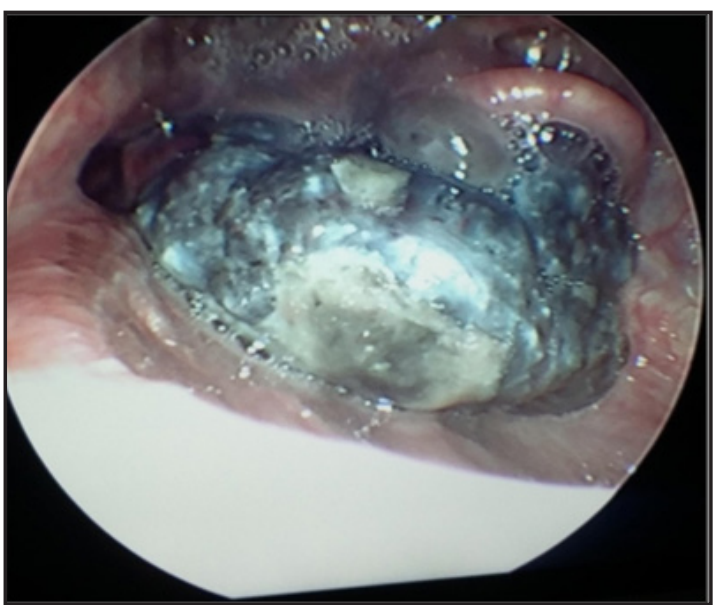

Fig.2. FOL showing the mass arising from laryngeal surface of epiglottis and Left A-E fold

had hyperchromatic nuclei, and they invaded both the mucosa and submucosa. Immunohistochemistry was positive for S-100, HMB-45 and Melan-A proteins. The tumour cells demonstrated a strong positive staining in both the nuclei and cytoplasm.(Fig. 4) The lesion was diagnosed as a primary malignant melanoma.

\section{Case 2: Neuroendocrine tumor presenting as a sinonasal} mass

A 45 year old female presented with bleeding from left nose and mild swelling of left naso-maxillary region for two months duration. On examination, she had deviated nasal septum to the right with pinkish mass in left nasal

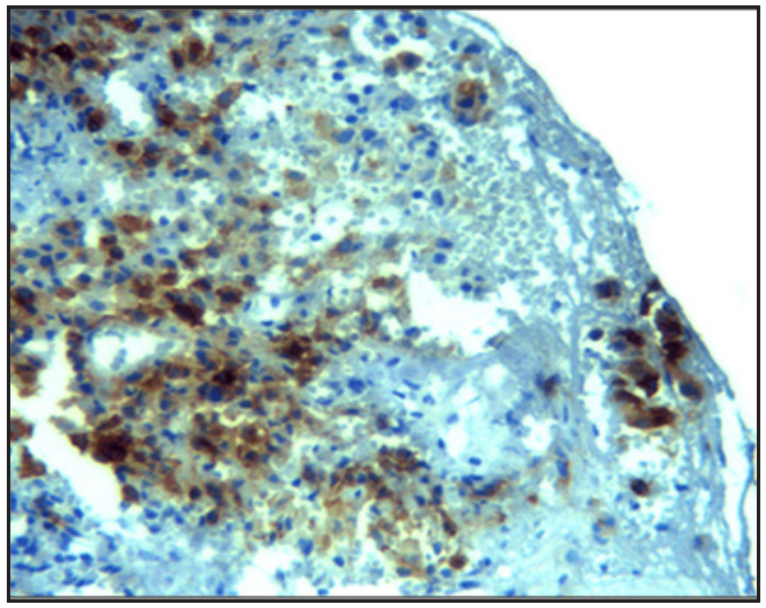

Fig. 4. Malignant melanocytes in the epithelium (Melan-A, 100×) 


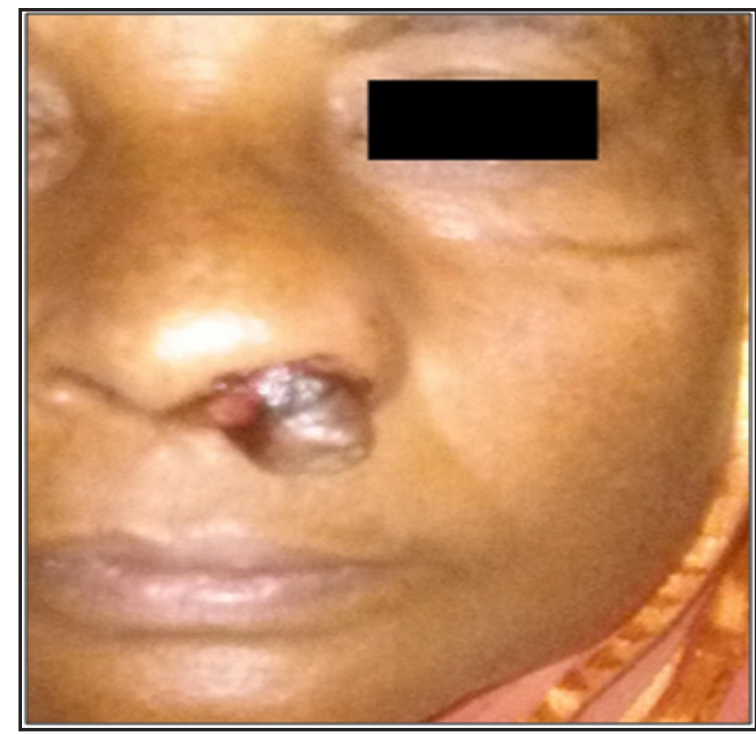

Fig. 5. A fleshy mass coming out of the left nostril

cavity protruding from the left nostril. (Fig. 5) Vision was normal. CT scan of nose and PNS revealed soft tissue mass occupying the entire left maxillary sinus and left ethmoids extending into and occupying the whole of the left nasal cavity. (Fig. 6) Our case falls into Kadish B stage. Biopsy from the nasal mass was reported as poorly differentiated neuroendocrine carcinoma.

As the biopsy report was neuroendocrine carcinoma

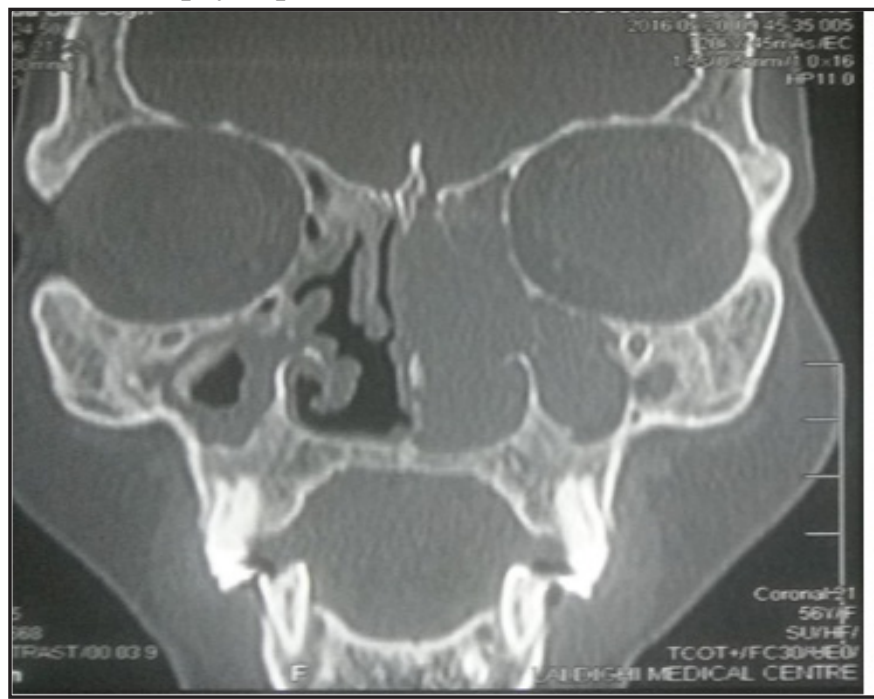

surgical treatment in the form left sided total maxillectomy followed by endoscopic clearance of left ethmoid was done and specimen sent for HPE. The histopathological report, was poorly differentiated small cell neuroendocrine carcinoma. Rosettes and necrosis were present along with brisk mitotic activity. (Fig. 7) Immunohistochemistry was positive for CK, synaptophysin and chromogranin. Radiotherapy and chemotherapy were administered to the patient. On follow-up patient is well with no recurrence even after eight months.

Immunohistochemistry always confirms the diagnosis of rare sinonasal malignancies. In the present case, synaptophysin and chromogranin were positive while S-100 was negative which favoured the diagonisis of neuroendocrine carcinoma over olfactory neuroblastoma.

Case 3: Rhinosporidiosis presenting as a cheek swelling A fifty-year-old male reported with complaints of swelling on the right side of cheek for three months, with a history of recent increase in its size. There was no history of trauma in the recent past and patient had no complaint of pain. On examination the nose, nasopharynx, oropharynx and eyes appeared normal. On local examination there was a single, soft to firm, discrete

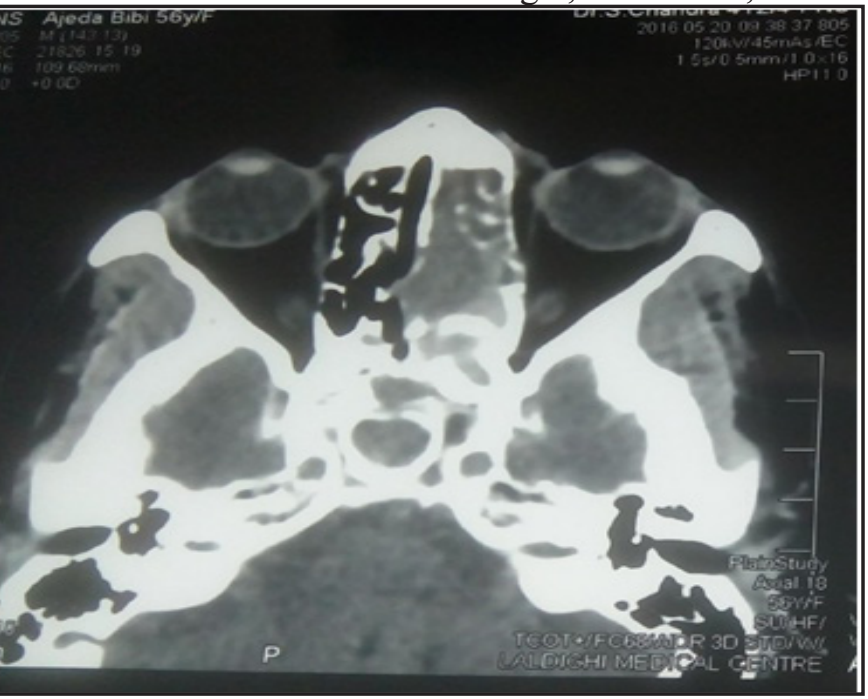

Fig. 6. CECT scan of nose and PNS showing the sinonasal mass 


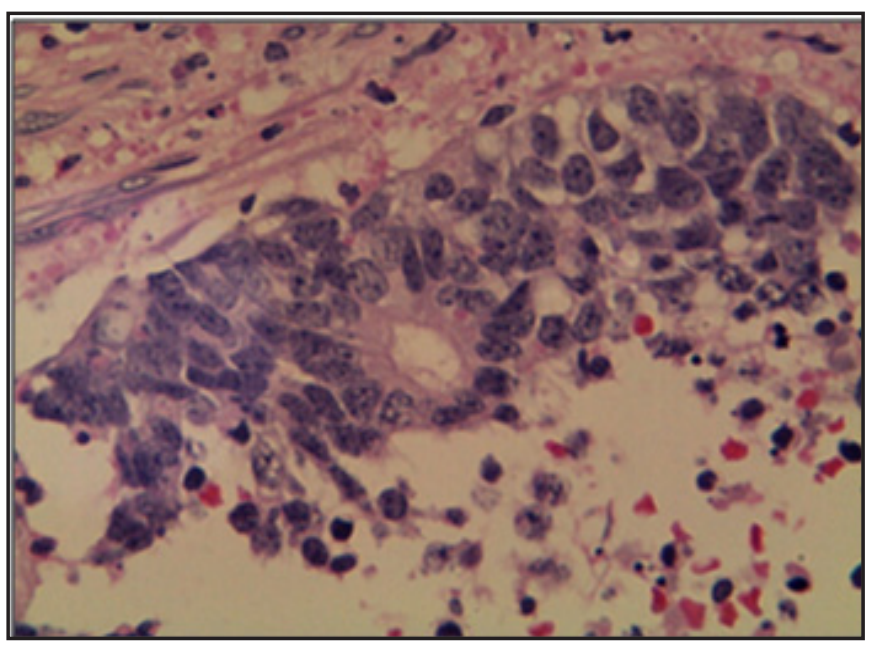

Fig. 7. Rosettes in NEC $(\mathrm{H} \& \mathrm{E} 40 \times)$

swelling on the right side of the cheek measuring about $5 \mathrm{~cm} \times 4 \mathrm{~cm}$. Overlying skin was normal in color and texture with no local rise of temperature. There was no tenderness felt on palpation with no fixity to overlying skin or underlying structure. On intraoral examination, it was seen that there was serous secretion coming from the inflamed opening of Stensen's duct and there was a fistulous opening in the right buccal mucosa. There was no cervical lymphadenopathy. USG showed a solidcystic lesion in the subcutaneous plane of size $3 \mathrm{~cm} x$ $1.5 \mathrm{~cm}$, with echogenic debris and internal septations. Surgical excision of the lesion was done under general anesthesia by intraoral route which included the distal margin of parotid duct and the fistula of the right buccal mucosa.

The excised specimen was submitted for histopathological examination. The Hematoxylin-Eosin stained section showed thin fibrocollagenous cystic wall lined by columnar to cubodial cells. Parts of the specimen showed the presence of numerous sporangia of R. seeberi. The diagnosis of rhinosporidiosis was primarily made by observing the distinctive morphologic features of R. seeberi in affected tissue.

\section{Discussion}

Malignant melanomas are malignant tumors of neural crest origin, arising from the melanocytes. Although most of them have cutaneous origin, a small number of melanomas occasionally arise from non-cutaneous tissue that contains melanocytes, such as leptomeninges, uvea and mucous membrane. Mucosal melanoma of the larynx is an extremely rare tumor with about 60 cases reported in the medical literature. . Mucosal melanomas although rare, are known to behave more aggressively and have less favourable prognosis compared to other melanoma subtypes. Most of mucosal melanomas occur in occult sites, which together with the lack of early and specific signs contribute to late diagnosis, and poor prognosis.

Neuroendocrine neoplasms are defined as epithelial neoplasms with predominant neuroendocrine differentiation. They can arise in almost every organ of the body although they are most commonly found in the gastrointestinal tract and respiratory system. In the nasal and paranasal sinus regions, squamous cell carcinoma is the most common tumor. Primary sinonasal neuroendocrine carcinomas are rare and represent a histological spectrum of differentiation. In contrast to other regions, neuroendocrine tumors of the sinuses have been reported to be recurrent and locally destructive. During the past 45 years, only 80 cases of small cell neuroendocrine carcinoma of nose and paranasal sinuses have been reported in literature.

Rhinosporidiosis is a benign chronic granulomatous disease most commonly affecting the nose, being quite rare in the cheek or the parotid duct. In our case patient had history of consumption of unprocessed well water. Surgical treatment of Rhinosporidiosis is excision of mass followed by cauterization of the base. . The life cycle of R. seeberi begins in the tissue as a spore, and it passes through several stages of development from trophocyte to juvenile sporangium to mature forms with changes in thickness and lamination of walls. The sporangia and sporangiospores of the organism can be seen with typical fungal stains such as Gomori Methenamine Silver (GMS), Periodic Acid-Schiff (PAS), and mucicarmine as well as with standard haematoxylin and eosin stain.

Treatment with the drug Dapsone appears to be promising which appears to arrest the maturation of the sporangia and to promote fibrosis in the stroma, when used as an adjunct to surgery. Recurrences are known to occur due to spillage of endospores on the adjacent 
tissue or incomplete excision.

\section{References}

1. Pantazapoulos PE. Primary malignant melanoma of the larynx. Laryngoscope 1964:74:95-102

2. Mackintosh JA. The antimicrobial properties of melanocytes, melanosomes and melanin and the evolution of black skin. J Theor Biol. 2001;211:101-13

3. Plonka PM, Passeron T, Brenner M, Tobin DJ et al. What are melanocytes really doing all day long...? Exp Dermatol. 2009;18:799-819
4. Mills E. Neuroectodermal neoplasms of the head and neck with emphasis on neuroendocrine carcinomas. Mod Pathol. 2002; 15: $264-78$

5. Babin E. Rouleau V. Marland O. et al. Small cell neuroendocrine carcinoma of the nasal cavity and paranasal sinuses. J Laryngol Otol. 2006; 120:289-97

6. Jain SN, Rao PVR. Rhinosporidiosis. Current Opinion in Otolaryngology and Head and Neck Surgery 1998; 6(3): 182-5

7. Sudarshan V, Gahine R, Daharwal A, Kujur $P$ et al. Rhinosporidiosis of the parotid duct presenting as a parotid duct cyst-a report of three cases. Indian $\mathrm{J}$ Med Microbiol. 2012;30(1):108-11. 